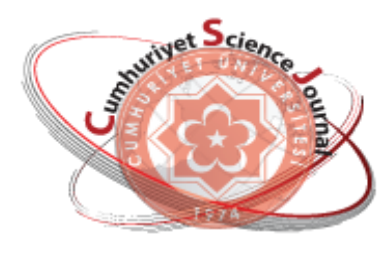

e-ISSN: 2587-246X

ISSN: $2587-2680$

\section{Cumburiyet Scionce Journal esy}

Cumhuriyet Sci. J., Vol.39-2(2018) 339-348

\title{
Lacewings Fauna of Çimen Mountain and Its Surroundings (Neuroptera: Planipennia) Kahramanmaraş, Turkey
}

\author{
Hakan BOZDOGAN \\ University of Ahi Evran, Department of Herbal and Animal Production, Technical Vocational School of \\ Kırşehir, Kırşehir, TURKEY
}

Received: 11.09.2017; Accepted: 19.03.2018

http://dx.doi.org/10.17776/csj.434317

\begin{abstract}
In this study, totally 146 specimens belonging to the Neuroptera Order (Neuroptera: Planipennia) were collected and evaluated in Çimen Mountain, between May and August in the years of 2015 and 2016. Totally, 16 species belonging to the Neuroptera Order have been determined. All of those species were recorded from Çimen Mountain for the first time. Among these species; Chrysopa perla (Linnaeus, 1758), Italochrysa italica (Rossi, 1970) and Palpares libelluloides (Linnaeus, 1764) are abundant and widespread species in the research area.
\end{abstract}

Keywords: Çimen Mountain, Kahramanmaras, fauna, biodiversity, neuroptera

\section{Çimen Dağı ve Çevresi Sinir Kanatlı Faunası (Neuroptera:Planipennia) Kahramanmaraş, Turkey}

Özet: Çimen Dağı ve çevresinden toplanan sinir kanatlı (Neuroptera: Planipennia) türlerinin değerlendirildiği bu çalışmada, 2015-2016 yıllarının Mayıs-Ağustos ayları arasında, toplam 146 birey toplanmıştır. Araştırma bölgesinde, Neuroptera takımına ait toplam 16 tür belirlenmiştir. Bu türlerin tamamı Çimen Dağı'ndan ilk defa kaydedilmiştir. Bulunan türlerden, Chrysopa perla (Linnaeus, 1758), Italochrysa italica (Rossi, 1970) ve Palpares libelluloides (Linnaeus, 1764) araştırma alanlarında sık rastlanan ve yaygın olan türlerdir.

Anahtar Kelimeler: Çimen Dağı, Kahramanmaraş, fauna, biyoçeşitlilik, sinir kanatlı

\section{INTRODUCTION}

Species belonging to Neuroptera, also known as lacewing, are the most widely observed predators which feed on nectar in ecosystems or harmful plants. Especially, Chrysopidae, Hemerobiidae and Coniopterygidae are used as biological control. Neuroptera Order live in large habitat extending from Melbourne, Australia in the West to Ontario and Queensland in the East. Neuroptera species are found in altitudes starting from seal level up to 2800 meters, mostly in needle-leaved and broad-leaved tree formations, in the front sides of stream or slack water and in various culture plants [1-3].
As their wings have a primary shape and lots of cross-lode, they are known as " dantela" or "sinir kanatlilar" in Turkish, "lacewing" in English and "Netzflugler" in German. Neuroptera (Planipennia) group has 687 species and 27 subspecies in Palearctic Area and are represented with more than 6500 species belonging to 17 families [4]. In Turkey, according to recent researches, there are 193 species and 6 subspecies belonging to 10 families (Ascalaphidae, Berothidae, Chrysopidae, Coniopterygidae, Dilaridae, Hemerobiidae, Mantispidae, Myrmeleontidae, Nemopteridae and Osmylidae) [5]. 
Natural or semi-natural unfinished areas create microhabitats in order for lacewings to leave eggs, to make cocon and to exhibit wintering behaviors. Besides that, they are found on most of broad-leaved tree formations, various culture lands, tropical fruit trees and some garden plants $[2,6]$.

There is few data about digestion behaviors of Hemerobidae. In intestine analysis, aphids' remnants are discovered and also few omnivores are found. Generally, they live in habitats where they will be able to find their prey. Investigations have shown that Hemerobius pasficus larvae need 350 various aphid species in order to become pupa.

Mountain ecosystem climates have various plants and wild animals and are complex and rich. Because of height difference, there are differences at short distance in terms of heat, light, wind and moisture. That is why mountain ecosystem is bio-diversity Island being gen bank, shelter for plants and animals and surrounded with habitats [7].

Altitudes around Cimen Mountain are between $440 \mathrm{~m}$ and $2.259 \mathrm{~m}$. The highest point of the area is Ulu Ziyaret Hill which is $2.259 \mathrm{~m}$. Cilmezar Hill $(1.889 \mathrm{~m})$ and Catalkaya Hill $(1.800 \mathrm{~m})$ are the other two highest points in the research area. There are three vegetation types which are forest, bush and step.

Forest Vegetation: This vegetation is found in different zones according to local climate condition, altitude ranges from $600 \mathrm{~m}$ to $1800 \mathrm{~m}$.

Brush vegetation: This vegetation type spreads around from $500 \mathrm{~m}$ to $900 \mathrm{~m}$.

Step vegetation: This vegetation type spreads in local areas which Abides and Cedrus forests that are ruined in $1600 \mathrm{~m}$ and above forest vegetation (1900-2250m) (Golluoglu, 2010). Cimen Mountains which take place in the West part of Amanos Mountains become habitat for various insect species including neuropteran. Regular zoning is seen in the area based on altitudes. Vegetation types can be ordered in that way:
Forest vegetation is between $600 \mathrm{~m}-1800 \mathrm{~m}$, brush vegetation is between $700 \mathrm{~m}-950 \mathrm{~m}$ and step vegetation is between $1600 \mathrm{~m}$ and $2100 \mathrm{~m}$ [8].

\section{METHOD}

\section{Study Area}

Research Area, Cimen Mountains, ranks among $36^{\circ} 30^{\prime} 00^{\prime \prime}-36^{\circ} 52^{\prime} 30^{\prime \prime}$ west longitude and $37^{\circ}$ $22^{\prime} 30^{\prime \prime}-37^{\circ} 37^{\prime} 30^{\prime \prime}$ north latitude whose distance is $45 \mathrm{~km}$ to Kahramanmaras city center. Size of the area is 17.522 ha. Uludaz Hill form is the highest point of Cimen Mountain by having $2.273 \mathrm{~m}$ altitude. Cimen Mountain is selected as research area because it includes many ecological species such as step, wetland and steppe. Survey was conducted in micro habitats and different altitudes in and around the Cimen Mountain between 2015 - 2016 years.

\section{Data Collection}

Specimens were collected by using different biotopes and altitudes by using net traps, electric lambs with charged and japan umbrella (Figure $1,2)$.

Samples were collected from short plants and weed through net traps whose radius is $20 \mathrm{~m}$ and thin: it was provided to fall them into fabrics which are covered under the tree previously by beating with stick like rolling pin from short, frequent, brush and thorn. Catching with japan umbrella carried out to collect species of Hemerobiidae and Mantispidae. As Neuroptera rest inside trees and big plants in hot and sunny time, catching insect process was conducted in morning or evening hours or dark shape areas that do not get sunlight in afternoon hours. To catch adult species, white fabric which is another method was used. 250-350 Watt lamb in the shape of screen and powered by a small portable generator hanged in $30 \mathrm{~cm}$ front of a cloth standing as centered. Specimens that came to light and alighted on curtain were collected with thin pin forceps. With the light trap, mostly Chrysopidae and Ascalaphidae species have been caught. 
Tajikistan, Turkmenistan, Ukraine, Old Yugoslavia, Greece [2, 4, 21-28].

Zoogeographic Origin: Pontomediterranean: Anatolia [2, 4, 11].

Family: Hemerobiidae Latreille, 1803

Hemerobius nitidulus Fabricius, 1777

Material Examined: 3 우 2 ถึं Kahramanmaraş, Gedikli Stream, 17.VI.2015

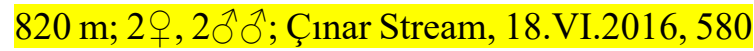
$\mathrm{m} ; 3+3 \hat{o}^{\lambda}$, Akyar Stream, 27.VI.2015, 1520m; 1 우, 1 ô $\hat{\jmath}$, Kuşharman Stream, 1526 m, 16.VIII.2016.

Turkey Distribution: Middle Anatolia Area, East Black Sea Area, Antalya, Ardahan, Aydın, Burdur, Denizli, Kars, Kocaeli, Kırklareli, Muğla, Sakarya [9, 18-20, 29-31].

World Distribution: Germany, Andora, Albania, Australia, Belgium, Bulgaria, England, Czech Republic, Denmark, Estonia, Finland, France, Croatia, Greece, Holland, Ireland, Spain, Sweden, Switzerland, Italy, Cambogia, Cyprus, Letonia, Liechtenstein, Lithuania, Luxemburg, Hungary, Mongolia, Norway, Poland, Romania, Russia, Siberia, Slovenia, Turkey, Ukraine [21, 22, 32-41].

Zoogeographic Origin: Siberian [2, 4, 11].

Hemerobius handschini Tjeder, 1957

Material Examined: $1 q q, \quad 5 \AA \precsim$; Kahramanmaraş, Hüsam Stream, 11.VII.2015, 977m; 2ᄋ, 1ठำ; Yavşan Stream, 14.VII.2015,

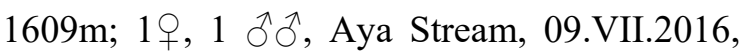
1123m; 4우우, 1 ふึ, Kelpınar Stream, 613 m, 11.VI.2016.

Turkey Distribution: Middle and South Anatolia, Antalya, Isparta, Denizli, Düzce, Kocaeli, Sakarya [18, 20, 31].
World Distribution: Germany, Australia, Bulgaria, Czech Republic, France, Croatia, Spain, Switzerland, İtaly, Hungary, Poland, Portugal, Romania, Slovenia, Turkey, Ukraine, Old Yugoslavia, Greece [2, 4, 23, 41-44].

Zoogeographic Origin: Holomediterranean $[2,4,11]$.

Hemerobius (Hemaerobius) stigma Stephens, 1836

Material Examined: $5 \%$ 우 $\quad 6 \hat{\partial} \delta^{\lambda}$; Kahramanmaraş, Ağıllı Stream, $621 \mathrm{~m}$, 17.VII.2015, 621m; 3우 5ðð; Fak1lar Stream, 09.VII.2016, $490 \mathrm{~m} ; 3$ ㅇ, 20ิ0َ, Kalekaya

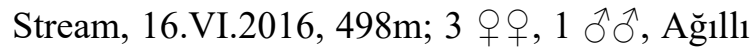
Stream, $621 \mathrm{~m}, 22$. VII.2016.

Turkey Distribution: North Anatolia [2].

World Distribution: Germany, Andora, Australia, Azor Islands, Bulgaria, Czech Republic, Denmark, Estonia, Morocco, Finland, France, Croatia, Holland, England, Spain, Sweden, Swetzerland, Japan, Canada, Canary Islands, Kazakstan, Cyprus, North Anatolia, Letonia, Liechtenstein, Lithuania, Luxemburg, Hungary, Mongolia, Norway, Poland, Portugal, Romania, Russia, Siberia, Slovenia, Ukraine, Yugoslavia $[2,4,10,16]$.

Zoogeographic Origin: Siberian -Nearctic [2].

Family: Myrmeleontidae Latreille, 1803

Palpares libelluloides (Linnaeus, 1764)

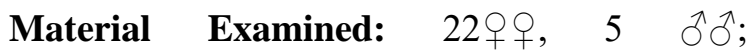
Kahramanmaraş, Yalangaz Stream, 17.VI.2016

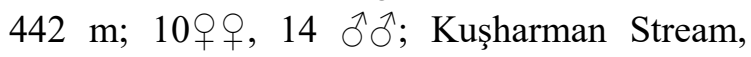
11.VI.2016, 1526 m; 12q, 22 ðૈ, Sazak Stream, 10.VII.2015, $1004 \mathrm{~m} ; 10$ 우, $3 \overbrace{}^{\widehat{\partial}}$, Çınarstream, $541 \mathrm{~m}, 16 . \mathrm{VI} .2016$.

Turkey Distribution: West, Middle, South and South West Anatolia. Aydın, Antalya, Denizli, Isparta, Muğla [13]. 
World Ditribution: Albania, BosniaHerzegova, Bulgaria, Algeria, Morocco, France, Croatia, Holland, Iraq, Iran, Spain, Italy, Caucasia, Cyprus, Hungary, Macedonia, Romania, Syria, Tunisia, Turkey, Jordan, Yugoslavia, Greece [4].

Zoogeographic Origin: Holomediterranean $[2,4,11]$.

Euroleon nostras (Geoffroy in fourcroy, 1785)

Material Examined: 1 웅 1 ถึ่ Kahramanmaraş, Karanlık Stream, 17.VII.2015, 1533m; 3ㅇ, 4 रิ ơ; Çamlı Stream, 17.VI.2015, 510m; 2ㅇ, 2 ふึ่, Dereboğazı, 11.VIII.2015, $1201 \mathrm{~m} ; 1$ 우우, 2 ふోळ, Karadere, 1228m, 16.VII.2015.

Turkey Distribution: Ankara, Iğdır, Sakarya $[9,19,31]$.

World Distribution: Germany, Australia, Azerbaijan, Belgium, Bosnia-Herzegova, Bulgaria, Sweden, Czech Republic, Denmark, Ermenia, Georgia, Greece, Morocco, France, Cloatia, Holland, Caucasia, Luxemburg, Spain, Sweden, Italy, Hungary, Poland, Romania, Russia, Liechtenstein, Slovakia, Slovenia, Turkey, Ukraine, old Yugoslavia $[2,4,21,23,26,27,38-40,45]$.

Zoogeographic Origin: Mediterranean [4].

Family: Chrysopidae Schneider, 1851

Dichochrysa venosa (Rambur, 1842)

Material Examined: 3 우, 2 ถึ่; Kahramanmaraş, Akyar Stream, 27.V.2009

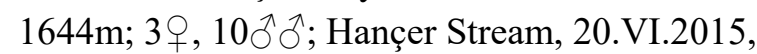

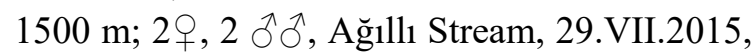
928m; 2우, 2 §ึग, Akyar Stream, 1644 m, 27.VI.2015.

Turkey Distribution: Mardin, Toros Mountains, Diyarbakır [3].
World Distribution: Afghanistan, Morocco, Turkey, France, Russia, Spain, Iran, Lebanon, Egypt, Pakistan, Portugal, Sudan, Saudi Arabia, Tunusia, Yemen [2,4].

Zoogeographic Origin: Pontomediterranean: Balkan [11].

Nineta carinthiaca (Hölzel, 1965)

Material Examined: 3 우, 2 ๙ึ; Kahramanmaraş, Ahmetçik Stream, 25.V.2015,

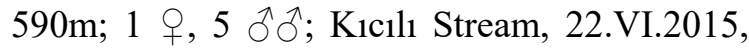

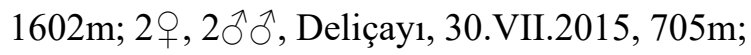
1우우, $2 \widehat{O}^{\widehat{\jmath}}$, Ağıllı Stream, 930m, 21.VI.2015.

Turkey Distribution: Ankara, North East Anatolia [3].

World Distribution: Australia, Hungary, Slovenia, Turkey $[2,4]$.

Zoogeographic Origin: Central European [11].

Chrysopa walkeri (McLachlan, 1893)

Material Examined: 3 우, 2 ふぇ; Kahramanmaraş, Y1k1kdeğirmendere,

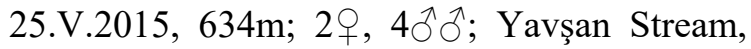
13.VI.2016, 1701m; 1 ㅇ, 2 ठิో , Aya Stream,

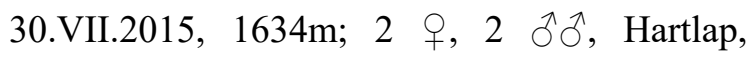
$1209 \mathrm{~m}, 26$. VI. 2015 .

Turkey Distribution: East Anatolia and Antalya [4,18,22].

World Distribution: Germany, Australia, Bulgaria, Czechoslovakia, Ermenia, France, Finland, Croatia, Spain, Sweden, Cyprus, Kirghizstan, Lebanon, Hungary, Mongolya, Moldova, Uzbekistan, Romania, Turkey, Ukraine, Yugoslavia [2,4].

Zoogeographic Origin: Siberian [11].

Italochrysa italica (Rossi, 1970) 
Material Examined: $13 ㅇ+$, $25 \hat{\partial} \hat{o}$; Kahramanmaraş, Kozlu Stream, 22.V.2015,

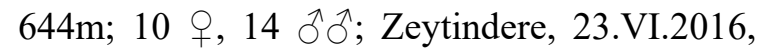

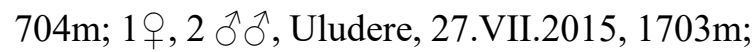
9우우, 11ठㅊ, Kelpinar Stream, $1044 \mathrm{~m}$, 24.VI.2015.

Turkey Distribution: Adiyaman, Ankara, Çanakkale, İzmir, Kahramanmaraş, Konya and Mersin [14,18].

World Distribution: Bulgaria, France, Iraq, Spain, Italy, Sweden, Lebanon, Egypt, Portugal, Romania, Turkey, Greece [2].

Zoogeographic Origin: Holomediterranean [11].
Brinckochrysa amseli (Hölzel, 1967)

Material Examined: 3 우, 2 ๙ิศ; Kahramanmaraş, Başkonuşaşkonusara Gavurdağ1 Village, 27.V.2015, $1482 \mathrm{~m} ; 1$ q, 4 §ô; Hançer Stream, 23.VI.2015, $1500 \mathrm{~m}$; 1 ㅇ,

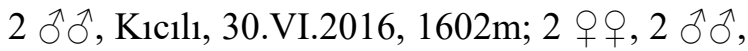
Gedikli, 590 m, 26.VI.2015.

Turkey Distribution: Adana [3].

World Distribution: Anatolia and Afganistan $[2,4]$.

Zoogeographic Origin: Holomediterranean [11].

Chrysopa perla (Linnaeus, 1758)

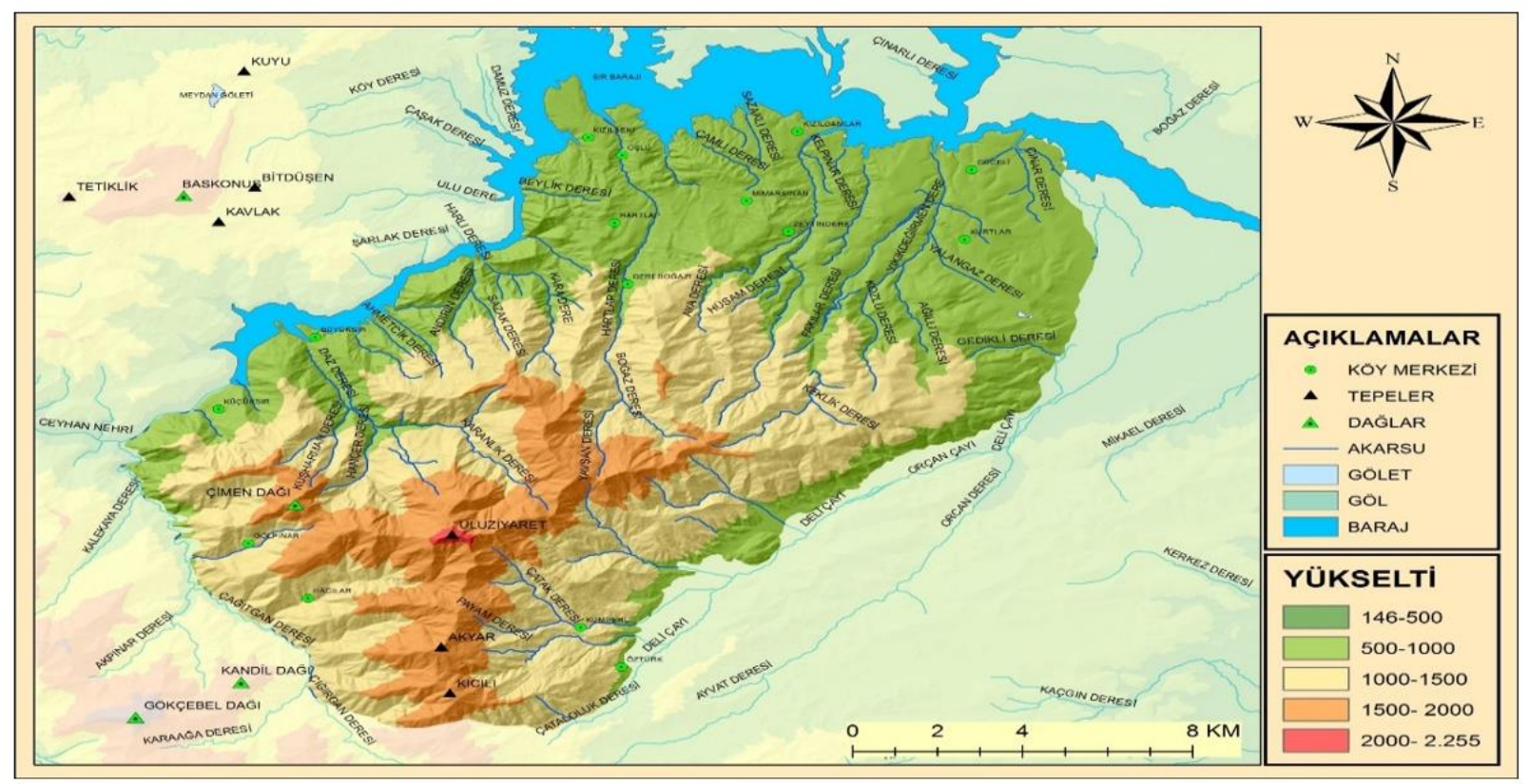

Figure 1. Çimen Mountain Map.

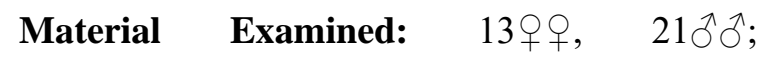
Kahramanmaraş, Kelpınar, 29.VIII.2016, 618m;

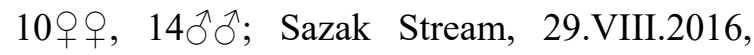

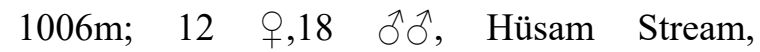

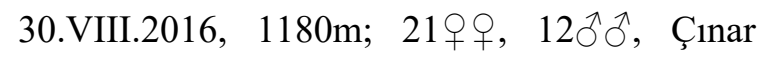
Stream, 541m, 14.VIII.2015.

Turkey Distribution: Ankara, İstanbul, Muğla and North Anatolia [2-4].
World Distribution: Germany, Australia, Belgium, Belarus, Bulgaria, BosniaHerzegovina, Czechoslovakia, Denmark, Ermenia, Estonia, Finland, France, Georgia, Holland, Spain, England, Switzerland, Sweden, Italy, Japan, Kazakhstan, Kirgizstan, Letonia, Luxemburg, Hungary, Malta, Norway, Poland, Romania, Russia, Siberia[2,4,41,46].

Zoogeographic Origin: Siberian [11]. 
Previous researches about $C$. septempunctata which is found in Uludaz, Cimen Mountain started to be conducted and meetings were done with people, and especially over 70 years old who live in Buyuk Sir Urban which is the closest residence place. It was founded that $C$. septempunctata which is known as "Nisan Bocegi" by urban people has existed more than 100 years in that area. Also, people in the Urban Area stated that there is increase on population of C. septempunctata as number of aphid is raised in July and August [47].

C. septempunctata is reported in mountains which have $1500 \mathrm{~m}$ altitude in France, Czechoslovakia, Poland, Greece and Japan. While Cimen Mountain, Uludaz Hill shows differences within its $2273 \mathrm{~m}$ altitude from the others, C. septempunctata which is in Vettore Mountain, Middle Italy and its highness is 2478 seems the same with that area [47].

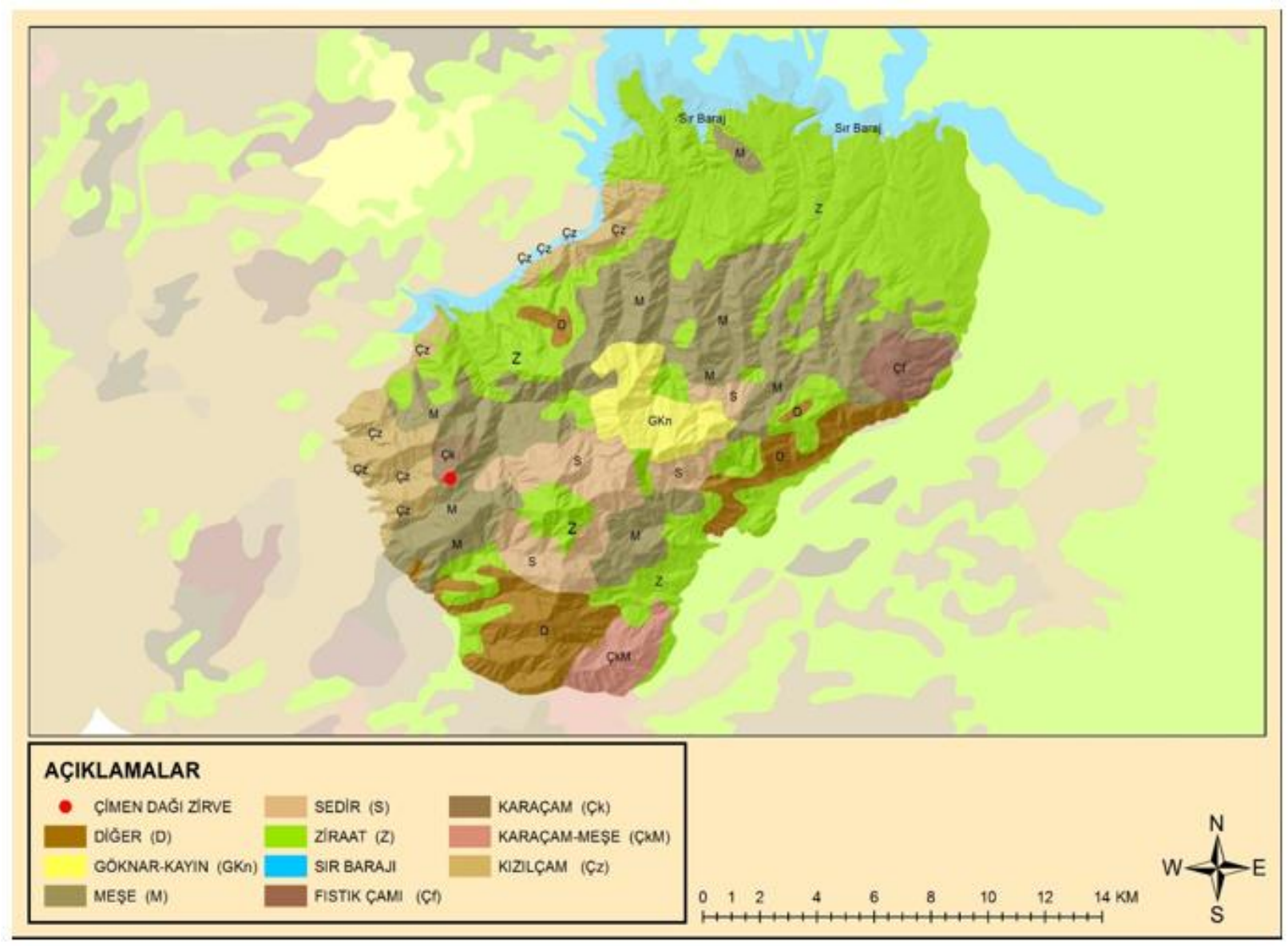

Figure 2. Çimen Mountain Altitude Scale Map.

\section{REFERENCES}

[1]. McEwen P.K., New T.R. and Whittington A., Lacewings in the crop Environments, Cambridge University Press, Cambridge, 2001; pp 1-546.

[2]. Aspöck H., Aspöck U. and Hölzel H., Die Neuropteren Europas. Eine zusammenfassende Darstellung der
Systematik, Ökologie und Chorologie der Neuropteroidea (Megaloptera, Raphidioptera, Planipennia) Europes, Goecke \& Evers, Krefeld, 1980, pp 1, 495.

[3]. Şengonca Ç., Systematic and taxonomic studies on Turkey Chrysopidae (Neuroptera) fauna. T.C Food, Agriculture and Livestock Ministry, 
General Directorate of Plant Protection and Quarantine Branch Directorate of Printing 1980; pp 1-138.

[4]. Aspöck H., Hölzel H. and Aspöck, U., Kommentierter Katalog der Neuropterida (Raphidioptera, Megaloptera, Neuroptera) der Westpaläarktis, Denisia 2001; pp 2-606.

[5]. Canbulat S., A checklist of Turkish Neuroptera with annotating on provincial distributions, Zootaxa 1552 (2007) 35-52.

[6]. Aydemir M. and Toros S., Erzincan İli Koşullarında fasulyelerde Zararlı Tetrnychus urticae Koch. (Acarina, Tetranyhidae)'nin doğal düşmanları. Türkiye 2. Biyolojik Mücadele Kongresi (26-29 Eylül 1990, Ankara), İzmir (1990) 261-27.

[7]. Küçükkaya İ., Türkiye Dağlık $\mathrm{Su}$ Havzalarının Genel Özellikleri ve Sürdürülebilir Yönetimi. Türkiye Dağları Birinci Ulusal Sempozyumu, Bildiriler Kitabı, Orman Bakanlığı, 183 (2002) 7781, Ankara.

[8]. Kısakürek Ş., Kahramanmaraş Çimen Dağı Örneğinde Dağlık Alan Yönetim Planlamas1, Ankara Üniversitesi, Fen Bilimleri Enstitüsü, Doktora Tezi, Ankara (2006).

[9]. Ari I., Aktas M. and Kiyak S., Notes on the Chrysopidae (Neuroptera) fauna of Ardahan, Igdir and Kars provinces of Turkey, Turkish Journal of Zoology, 31 (2007) 201-208.

[10]. Aspöck H. and Hölzel H., The Neuropteroidea of North Africa, Mediterranean Asia and of Europe: a comparative review (Insecta). pp. 31-86 in Canard, M.; Aspöck, H., and Mansell, M. W. (eds.). Pure and Applied Research in Neuropterology. Proceedings of the Fifth International Symposium on Neuropterology (2-6 May 1994, Cairo, Egypt). Privately printed, Toulouse, France, (1996). pp 1-341.

[11]. Popov A., and Letardi A., Comparative zoogeographical analysis of Neuropterida of the Apennine and Balkan peninsulas.
In: Proceedings of the Tenth International Symposium on Neuropterology (Eds., D. Devetak, S. Lipovšek and A.E. Arnett), Maribor, (2010) pp. 239-256. 20

[12]. Aspöck H. and Aspöck U., Die Neuropteren Mitteleuropas: ein Nachtrag zur "Synopsis der Systematik, Ökologie und Biogeographie der Neuropteren Mitteleuropas." Naturkundliches Jahrbuch der Stadt Linz, (1969) 17- 68.

[13]. Şengonca Ç., Beitrag zur Neuropteren fauna der Türkei, Nachrichtenblatt der Bayerischen Entomologen, 28-1 (1979) 10-15.

[14]. Kıyak S. and Özdikmen H., Über Einige Neuropterenarten Von Soğuksu Nationalpark (Kızılcahamam, Ankara), Priamus, 6(3/4) (1993) 156-160.

[15]. Kacirek A., Beitrag zur Kenntnis der Familien Myrmelontidae, Ascalaphidae and Nemopteridae (Neuroptera) der Türkei. Klapalekiana, 34 (1998)183-188.

[16]. Canbulat S. and Özsaraç Ö., Çiçekdağı (Kırşehir) Neuropterida Faunası (Insecta; Neuroptera, Raphidioptera), Türkiye XVI. Ulusal Biyoloji Kongresi, İnönü Üniversitesi, Fen-Edebiyat Fakültesi, Biyoloji Bölümü, Malatya, (2002) 4-7 Eylül.

[17]. Canbulat S. and Kiyak S., Four species of Lacewing (Insecta; Neuroptera) new to the fauna of Turkey, Zoology in the Middle East, 32 (2004) 113-114.

[18]. Canbulat S. and Kiyak S., Contribution of the Fauna of Neuroptera (Insecta) of South-Western Anatolia. Annals of the Upper Silesian Museum Entomology, 13 (2005) 9-60.

[19]. Arı İ., Aktaş M. and Kıyak S., Notes on the Chrysopidae (Neuroptera) Fauna of Ardahan, Iğdır and Kars Provinces of Turkey, Turkish Journal of Zoology, 31 (2007) 201- 208.

[20]. Berber A., Samanlı Dağları (Geyve Boğaz1 bat1s1) Neuroptera (Insecta) išpejfaunasının araştırılması. Yüksek Lisans Tezi. Sakarya Üniversitesi. Fen Bilimler iscep:Enstitüsü (2008). Sakarya. 
[21]. Popov A., Wissenschaftliches Ergebnis der zoologischen Expedition des Nationalmuseums in Prag nach der Türkei. Raphidioptera, Neuroptera und Mecoptera. Acta Entomologica Musei Nationalis Pragae, 39 (1977) 271-277.

[22]. Popov A., Prinos kum izuchavaneto na mrezhokrilite nasekomi (Neuroptera) na ispivitosha [=Contributions to the knowledge of the Neuroptera of the Vitosha istepmountains]. Fauna na Iugozapadna Bulgaria, 3 (1990) 78-87,

[23]. Ábrahám L., and Papp Z., A Matra Muzeum Neuropteroidea gyujtemenye (Planipennia, Megaloptera, Raphidioptera, Planipennia), Folia Historico Naturalia Musei Matrensis, 14 (1989) 81-86.

[24]. Sziráki Gy., Ábrahám L., Szentkirályi F. and Papp Z., A check-list of the Hungarian Neuropteroidea (Megaloptera, Raphidioptera, Planipennia). Folia Entomologica Hungarica, 52 (1992) 113118.

[25]. Ábrahám L. and Sziraki G., A BedaKarapancsa Tajvedelmi Körzet recesszarnyu faunajanak termeszetvedelmi ertekelese (Megaloptera, Raphidioptera, Neuroptera), Dunantuli Dolgozatok A Termeszettudomanyi Sorozat, 6 (1992) 71-78.

[26]. Pantaleoni R.A. and Letardi A., I Neuropterida della collezione dell'Istituto di Entomologia "Guido Grandi" di Bologna. Bollettino dell'Istituto di Entomologia "Guido Grandi" dell'Università di Bologna, 52 (1998) 1545.

[27]. Hölzel H. and Wieser C., Die Netzflügler Kärntens. Eine zusammenfassende Darstellung der Autökologie und Chorologie der Neuropterida (Megaloptera, Raphidioptera, Neuroptera) Kärntens. Carinthia II: Mitteilungen des Naturhistorischen Landesmuseums für Kärnten, 189-109 (1999) 361-429.
[28]. Mirmoayedi A., New species of Iranian neuropterans, In: Proceedings of the 13th Plant Protection Congress of Iran (Eds. Hojat, H.), Agricultural teaching institute (Amuzeshkadeh Keshavarzi) Karadj, 1998; pp 1-250, Iran.

[29]. Háva J., The genus Deleproctophylla Lefebvre, 1842 (Insecta: Neuroptera: Planipennia: Ascalaphidae) from the collection of the Department of Entomology, National Museum Praha. Casopis Narodniho Muzea Rada Prirodovedna, 169(1-4) (2000) 1-16.

[30]. Onar N., Trakya bölgesi Neuroptera faunası üzerine taksonomik ve faunistik araştırmalar, Trakya Üniversitesi, Fen Bilimleri Enstütüsü, Doktora Tezi (2007) ss 136 Edirne. istep:

[31]. Özcan G., Samanlı Dağları (Geyve Boğazı Doğusu) Neuroptera (Insecta) Faunasının Araştırılması Sakarya Üniversitesi, Yüksek Lisans Tezi, Fen Bil. Enstitüsü, 2008; ss 151 Sakarya.

[32]. Hölzel H., Die Netzflüger Kärntens 1. Nachtrag, Carinthia II, 163-83 (1973) 497-506.

[33]. Popov A., Coniopterygiden aus Bulgarien (Neuroptera), Ent. Nachr. Berichte 30 (1986) 167-171.

[34]. Popov A., Baum-und strauchbewohnende Neuropteren in Bulgarien, Acta Zoologica Bulgarica, (1991) 41:26-36.

[35]. Monserrat V.J., Contribucion al conocimiento de los Neuropteros de Orense (Neu. Planipennia), Boletín de la Asociacion Española de Entomologia, 2 (1978) 169-184.

[36]. Monserrat V.J., Contribucion al conocimiento de los Neuropteros de Italia (Neuroptera, Planipennia), Neuroptera International, 1 (1980a) 48-64.

[37]. Monserrat V.J., Contribucion al conocimiento de los Neuropteros de Toledo (Neuroptera, Planipennia), Graellsia, 34 (1980b) 177-193.

[38]. Monserrat V.J., Contribucion al conocimiento de los neuropteros de Salamanca (Neu. Planipennia), Revista 
Espanola de Entomologia, 59 (1984) 165177.

[39]. Monserrat V.J., Sinoposis de los hemerobidos de la Peninsula Iberica (Neuroptera, Planipennia), Actas de las VIII Jornadas Asociación Española de Entomología (1986) 1200-1223.

[40]. Monserrat V., Sobre los Neuropteros ibericos (IV) (Neur.), Boletin Asoc. esp. Entom., 10 (1986) 95-105.

[41]. Abrahám L. and Papp Z., Myrmeleon bore (Tjeder, 1941) in Hungary (Planipennia, Myrmeleontidae).

Neuroptera International 6 (1991) 137-139.

[42]. Ábrahám L., Adatok Az Alpokalja Neuropteroidea

Faunajahhoz

(Megaloptera, Raphidioptera es Planipennia), Savaria A vas Megyei Muzeumok Ertesitoje, 20-2 (1992) 23-39.

[43]. Ábrahám L., Natural protection studies on the neuropteroids (Megaloptera, Raphidioptera, Neuroptera) fauna of the Duna-Dráva National Park, II, Dunantuli Dolgozatok A Termeszettudomanyi Sorozat, 9 (1998) 269-289.
[44]. Sziráki G., Ábrahám L., Szentakirályi F. and Papp Z., A Check-list of the Hungarian Neuropteroidea (Megaloptera, Raphidioptera, Planipennia), Folia Entomologica Hungarica, 52 (1992) 113119.

[45]. Aspöck H. and Hölzel H., The Neuropteroidea of North Africa, Mediterranean Asia and of Europe:a comparative review (Insecta), Pure and Applied Research in Neuropterology, Proceedings of the Fifth International Symposium on Neuropterology, Canard, M., Aspöck, H., Mansell, M.W (1996) 31-86.

[46]. Greve L. and Kobro S., Preliminary data on abundance of phototactic Neuroptera and Rephidioptera in SE Norway as indicated by light-trap catches, Acta Zoologica Fennica, 209 (1998) 119-120.

[47]. Ricci C., Ponti L. and Pires A., Migratory Flight and Prediapause Feeding of Coccinella septempunctata (Coleoptera) Adults in Agricultural and Mountain Ecosystems of Central Italy. Eur. J. Entomol. 102 (2005) 531-538. 